
-

$\longrightarrow$

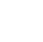




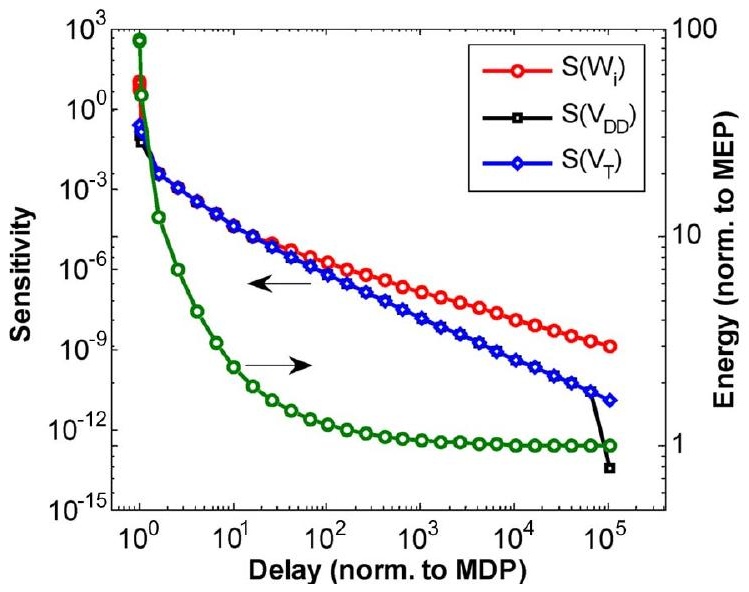

Fig. 4. Energy-delay sensitivity $S(x)=(\partial E / \partial x) /(\partial D / \partial x)$ to sizing $\left(W_{i}\right)$ $\left(V_{D D}\right) \quad\left(V_{T}\right) \quad y$-axis), and energy-delay trade-off (right $y$-axis) for a 32-bit carry look-ahead adder.

In (10) and (11), $\alpha$ represents the activity factor of the datapath, or the average activity for all gates. $K_{s w}$ and $K_{l k}$ represent technology (and fitting) constants. N ote that the energy-per-operation $E_{\mathrm{op}}$ is the path energy $E$ divided by the activity factor $\alpha, E_{\mathrm{op}}=E / \alpha$. As activity approaches zero, $E_{\text {op }}$ would approach infinity. This may seem counterintuitive at first, but makes sense because no operation is performed at zero activity yet (leakage) energy is being dissipated. Separation of voltage- and sizedependent parameters in (11) will prove useful in the derivative analysis, discussed next.

\section{SENSITIVITY ANALYSIS}

In this section, we present a framework to analyze the impact of gate sizing, supply and threshold voltage on energy-delay trade-offs. The energy-delay trade-offs via voltage and gate sizing will be quantified using the concept of energy-delay sensitivity. The sensitivity to a parameter $x$ represents a percent reduction in energy for a percent increase in delay, $S(x)=(\partial E / \partial x) /(\partial D / \partial x)$, [1], [31], [32]. Previous work [1], [32] has shown that sizing was the most effective around MDP. Here, the emphasis will be placed on the trade-offs around MEP. Let's examine the sensitivities of the optimization parameters along the optimal energy-delay (E-D) curve.

Fig. 4 shows simulated energy-delay sensitivity for an adder as well as optimal E-D trade-off when gate sizing, supply and threshold voltage are varied. Fig. 5 shows a closer look into areas around M DP [Fig. 5(a)] and M EP [Fig. 5(b)] to compare techniques for high-performance and lowenergy design optimization. On the optimal E-D curve, the sensitivities of the active parameters are equal. Lower sensitivity represents more delay reduction for a fixed energy increase or less increase in energy for a fixed delay reduction. When the sensitivity to a parameter deviates from the lowest curve, such parameter has reached its constraint limit, and is no longer active to support further energy reduction. This is the case with $V_{T}$ and sizing $\left(W_{i}\right)$ at MEP [Fig. 5(b)], and $V_{T}$ and $V_{D D}$ at M DP [Fig. 5(a)]. As expected, near MEP, $V_{D D}$ adjustment has the lowest sensitivity (it has least increase in energy for a given delay reduction), and thus the most effective parameter in delay reduction. Notice that we are looking at energy-delay sensitivity. Delay-energy sensitivity (as a measure of delay improvement for a given energy increase) to $V_{D D}$ would be the highest, just like E-D sensitivity to sizing is the highest around MDP. As we traverse up the E-D curve, from Fig. 5(b) to Fig. 5(a), $V_{T}$ also becomes significant, while sizing becomes significant only for high- $V_{D D}$ and low- $V_{T}$ scenarios, as we move towards high-performance regime in Fig. 5(a).

Sensitivity formulas (12)- (14), obtained from the delay and energy models from Section II, can be used to analytically calculate results from Figs. 4 and 5. Partial

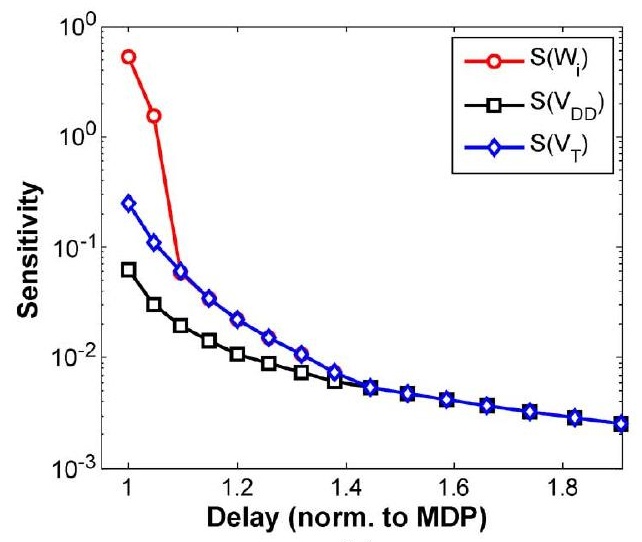

(a)

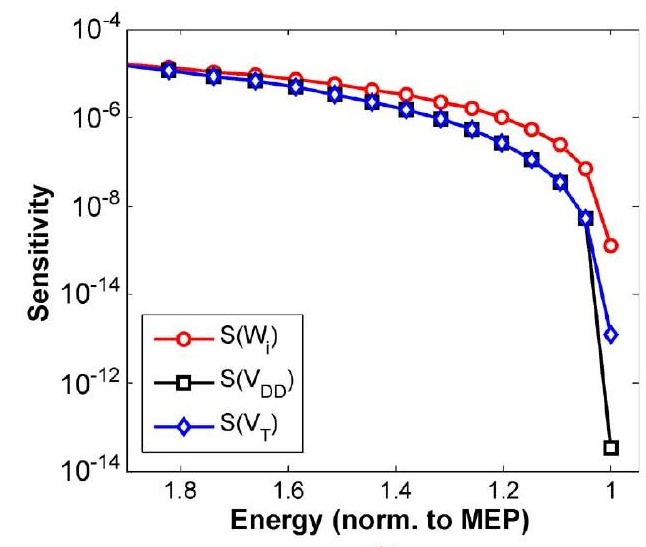

(b)

Fig. 5. Energy-delay sensitivity $S(x)=(\partial E / \partial x) /(\partial D / \partial x)$ near (a) MDP and (b) MEP for a 32-bit carry look-ahead adder from Fig. 4. 
derivatives with respect to $V_{D D}, V_{T}$, and $W_{i}$ lead to the following sensitivity results:

$$
\begin{aligned}
S_{V_{D D}}= & \frac{\partial E / \partial V_{D D}}{\partial D / \partial V_{D D}}=\frac{E_{s w}}{D} \cdot \frac{2}{1-N_{0}}+\frac{E_{l k}}{D} \\
& \frac{2+\sigma \cdot \frac{V_{D D}}{n \cdot \phi_{t}}-N_{0}}{1-N_{0}} \\
N_{0}= & \frac{1+\sigma}{\sqrt{I C}} \cdot \frac{V_{D D}}{n \cdot \phi_{t}} \\
S_{V_{T}}= & \frac{\partial E / \partial V_{T}}{\partial D / \partial V_{T}}=\frac{E_{l k}}{D} \cdot(1-\sqrt{I C}) \\
S_{W_{i}}= & \frac{\partial E / \partial W_{i}}{\partial D / \partial W_{i}}=\frac{e c_{i}}{K_{d} \cdot \frac{V_{D D}}{I C} \cdot\left(f_{i-1}-f_{i}\right)}+\frac{E_{l k}}{D} \\
& +\frac{E_{l k}}{K_{d} \cdot \frac{V_{D D}}{I C} \cdot\left(f_{i-1}-f_{i}\right)}
\end{aligned}
$$

where $f$ represents the effective fanout $f=g \cdot h$ for a gate.

To demonstrate the sensitivity of $V_{D D}$ and $W_{i}$ in the E-D space, Fig. 6 plots energy-delay optimization space when $V_{D D}$ and $W_{i}$ are individually tuned, starting from MEP. As predicted, scaling $V_{D D}$ is much more effective than using $W_{i}$ around MEP, because more delay improvement is possible for a given increase in energy. Actually, sizing is hardly effective until we get close to MDP.

Therefore, unlike MDP where sizing was the most dominant optimization variable, supply voltage should be used around M EP. This is because at M EP leakage current/ energy is linear function of $W_{i}$ and so is performance, while $V_{D D}$ is more effective for performance increase than sizing because $V_{D D}$ exponentially affects performance. Given the large disparity in sizing and supply sensitivities, we may reduce sizing (if possible) around MEP to create

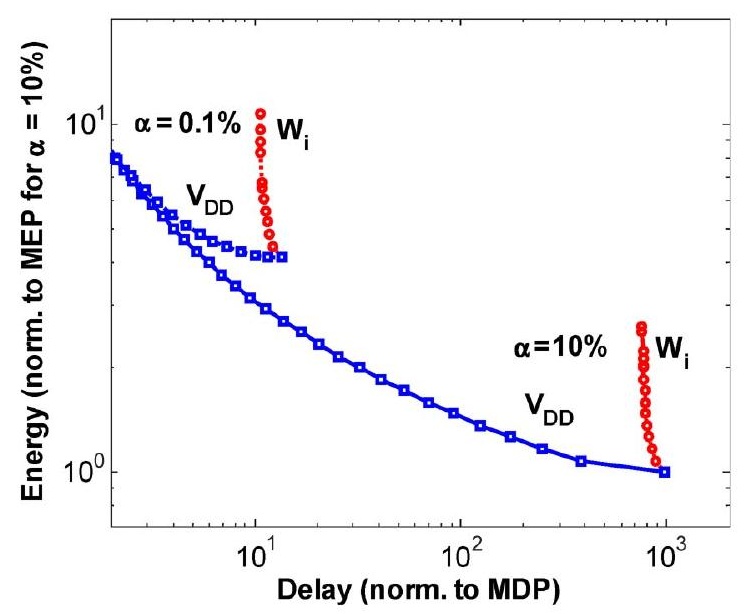

Fig. 6. Energy-delay trade-off after gate sizing $\left(W_{i}\right)$ and voltage scaling $\left(V_{D D}\right)$ for different activity levels for a 32-bit carry look-ahead adder from Fig. 4. energy slack that can be utilized by a small increase in $V_{D D}$ for overall performance increase. This is similar, albeit in different order of adjusting variables, to increasing $V_{D D}$ around MDP to create timing slack that can be utilized by sizing for overall energy reduction [1]. These trade-offs are generally not possible at MEP/M DP since the sizing and supply variables reach their bounds at these extreme points, so the use of sizing (MDP) or $V_{D D}$ (MEP) is the most optimal. Indeed, this is really good news for MEP region, because supply adjustment is easier to do than to adjust gate sizing. Gate sizing involves many more variables than simple $V_{D D}$ scaling. Besides, global $V_{D D}$ scaling does not require any layout changes and could be done after chip fabrication.

\section{ENERGY-DELAY OPTIMIZATION}

Most practical systems involve supply and sizing optimization, while threshold is selected from the available discrete values. This section explores supply and sizing optimizations for low- and high- $V_{T}$ devices to compare options offered by the two thresholds. The optimization will then be expanded to include $V_{T}$, which can be performed at the device level (e.g., body-bias) and at the circuit level (e.g., type of logic family).

We start the optimization from MEP as a reference. Unlike M DP, which is a fixed point in the E-D space, MEP depends on circuit activity. Let's then first examine M EP as a function of activity factor and $V_{T}$. The discussion below is based on the 32-bit carry look-ahead adder example.

Plots in Fig. 7 show MEP and IC versus activity for high- and low- $V_{T}$ designs. Since M EP is leakage-limited, HVT will always yield lower energy at the same activity. Under a very low activity factor, total energy of the circuit is dominated by its leakage energy, therefore the high- $V_{T}$ cells gain significant advantage for low activity factors. For activity factor of $0.01 \%$, for example, MEP of the HVT design achieves a 10-times lower leakage energy compared to the LVT design. Even under a high-activity factor of $10 \%$, M EP of the HVT design is still lower in energy than that of the LVT design. It is also interesting to observe that IC corresponding to MEP greatly varies with the activity factor. For $\alpha=0.1 \%, I C=5$ minimizes energy for low- $V_{T}$ devices, while for $\alpha=10 \%$, M EP occurs around IC $=0.03$ [Fig. 7(b)]. MEP is important, because it is the starting point in our optimizations. The plots in Fig. 7 do not indicate performance, which must be considered for a complete E-D comparison.

Optimal energy-performance trade-off of the same adder is shown in Fig. 8, along with the corresponding IC and $V_{D D}$ curves in Fig. 9. From the E-D plot in Fig. 8, it is evident that al though high- $V_{T}$ cells achieve lower energyper-operation than low- $V_{T}$ cells, HVT has 10- to 100-times lower performance than LVT. Such large performance penalty for marginal energy reduction is highly undesirable in ULP design. For performance-constrained low-power 



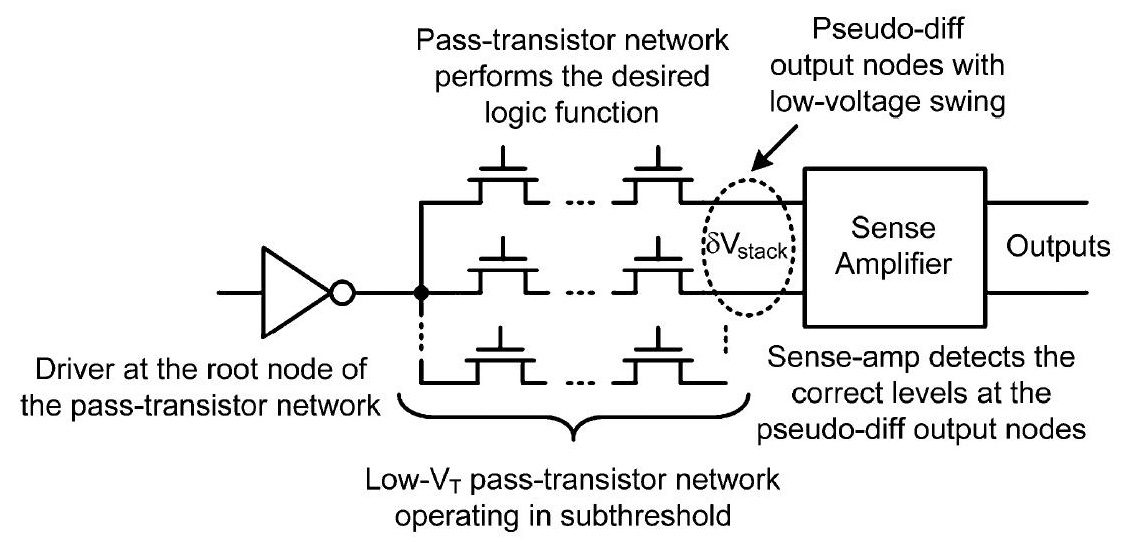

Fig. 12. Sense-amplifier-based pass-transistor logic (SAPTL) basic architecture.

the energy needed by the sense amplifier to resolve the correct stack output becomes too large. Typically, a $\delta V_{\text {stack }}>100 \mathrm{mV}$ is easily achievable at $V_{T, \text { stack }} \approx 100 \mathrm{mV}$ and can be detected with reasonable sense-amplifier energies, allowing the pass-transistors to operate comfortably in the $V_{T, \text { stack }}+\Delta V$ region.

Since $V_{T, \text { stack }}$ is different from the sense amplifier and driver threshold voltages, where leakage dominates at very low energy levels, operation in the near- or below- $V_{T}$ region is desirable. One possible relation between threshold and supply vol tages for the different components of the SAPTL is illustrated in Fig. 13. The pass-transistor stack has a threshold voltage $V_{T, \text { stack }}$ below the nominal $V_{T}$ of logic. Stacking is the key factor for leakage control thus allowing for this configuration of logic gates.

The SAPTL delay can be expressed as the sum of the sense amplifier and driver delays, $D_{\text {active }}$ and the stack delay, $D_{\text {stack. }}$. Assuming a simple dominant-pole model for the pass-transistor network, $D_{\text {stack }}$ can be expressed as:

$$
D_{\text {stack }}=\frac{k_{1} \cdot n_{\text {depth }}^{2}}{V_{D D}-V_{T, \text { stack }}}
$$

where $n_{\text {depth }}$ is the depth of the pass-transistor network, i.e., the number of transistors traversed by the signal injected from the root to the output, and $k_{1}$ is a constant.

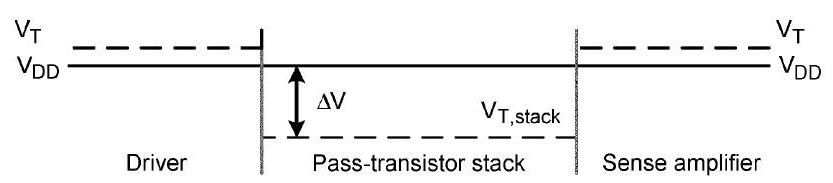

Fig. 13. One possible SAPTL supply and threshold voltage scenario showing subthreshold operation in the sense amplifier and driver and above-threshold operation in the pass-transistor stack.
Thus, we can express the total SAPTL delay over $M$ identical stages as:

$$
D_{\text {SAPTL }}=M \cdot D_{\text {active }}+M \cdot \frac{k_{1} \cdot n_{\text {depth }}^{2}}{V_{D D}-V_{T, \text { stack }}} .
$$

Note that if the delay of the stack dominates, then reducing $V_{T, \text { stack }}$ is an effective way of reducing the delay.

The energy required by the SAPTL for a single operation is thus:

$$
\begin{aligned}
E_{S A P T L} & =M \cdot C \cdot V_{D D}^{2}+M \cdot V_{D D} \cdot \sum_{i=1}^{n_{\text {depth }}} V_{i} \cdot C_{i} \\
& +V_{D D} \cdot I_{\text {leak }} \cdot M^{2} \cdot\left(D_{\text {active }}+\frac{k_{1} \cdot n_{\text {depth }}^{2}}{V_{D D}-V_{T, \text { stack }}}\right) .
\end{aligned}
$$

The first two terms of (17) represent the active energy used by the sense amplifier and driver. Note that the voltage swing of the internal stack nodes can be kept well below $V_{D D}$. The last term represents the leakage energy due to both the driver and sense amplifier.

From (17), we can see that as $V_{T, \text { stack }}$ is reduced, the leakage energy is also reduced. In practice however, this increases the current flow in the off-path stack capacitances, and thus leads to a corresponding increase in off-path node voltages, which tends to cancel-out any energy reduction, but still allowing delay improvement. If we assume that for a certain logical operation, $n_{\text {depth }} \cdot M$ is a constant, i.e., it can be implemented using either many shallow SAPTL stacks or very few but deep stacks, we can then see that stack complexity and gain can be traded off against each other to achieve a desired energy-delay operating point.

In order to understand how various logic functions are implemented, consider the pass-transistor stack that implements a 4-input XOR function as shown in Fig. 14. 
Fig. 14. A 4-input SAPTL XOR showing the pass transistor stack structure where each circle represents an NMOS transistor controlled by the corresponding input variable.

Each path from the root of the stack to $S$ represents a minterm and each path from the root to $S$ represents a maxterm. It can be observed from Fig. 14 that the SAPTL implementation of XOR gates is very straightforward. By increasing the complexity of the stack, in this case increasing the number of inputs to the XOR gate, the sense amplifier and driver overhead per input can be reduced, at the expense of decreased performance. This can be seen in Fig. 15, where the energy and delay of a 6 input and 16-input SAPTL XOR gate are compared to their static CM OS equivalents. With the same $V_{T}$ (equal to low$V_{T}$ ), SAPTL reduces energy below MEP of CMOS due to longer stacks (higher effective $V_{T}$ ) and lower leakage.

The capability of SAPTL to decouple $I_{\text {Leakage }}$ and $V_{T, \text { stack }}$ is illustrated using a self-timed 64-byte parallel CRC16 generator (as used in error detection). The threshold voltages of the pass-transistors (implemented using low- $V_{T}$ devices) are reduced using varying degrees of forward body biasing. The simulated results are shown in Fig. 16 with supply voltage and activity as independent parameters. The simulation results show that the overall circuit delay can be reduced with almost no impact on energy even at low activity factors such as $\alpha=1 \%$. These results are constrained by the limited effectiveness of body biasing as a means to control $V_{T, \text { stack. }}$ The availability of devices with even lower threshold would be desirable as it would increase the effectiveness of SAPTL for energy reduction.

As can be seen in Fig. 16, the performance improvement through body biasing is more prominent at the higher supply voltage $\left(V_{D D}=0.5 \mathrm{~V}>V_{T}\right)$, at which the delay of the stack dominates the total delay. At lower supply voltages $\left(V_{D D}=0.3 \mathrm{~V} \approx V_{T}\right)$, the delay of the sense amplifier as well as the hand-shaking circuitry [15] dominates since it is near the edge of subthreshold operation, limiting the performance gains obtainable through reduction of the $V_{T, \text { stack. }}$. Circuit- and logic-level techniques are foundation for architecture-level optimizations, which will be next discussed in Section VI.

\section{ARCHITECTURAL OPTIMIZATION}

Just as parallelism showed to be effective for energy reduction around MDP, time-multiplexing is best suited for performance increase around MEP. Architectural

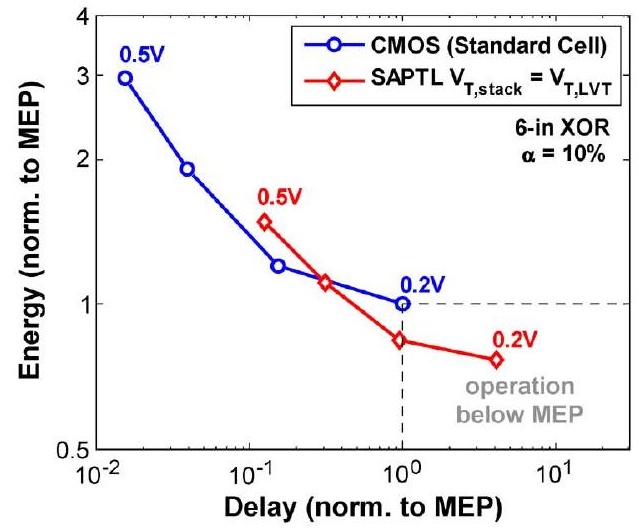

(a)

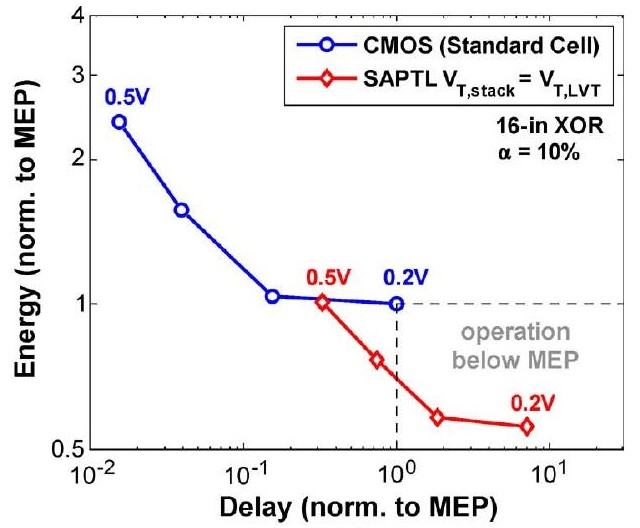

(b)

Fig. 15. Energy-delay characteristics of SAPTL designs: (a) 6-input XOR, (b) 16-input XOR. The plots show operation below MEP of static CMOS designs. 
Fig. 14. A 4-input SAPTL XOR showing the pass transistor stack structure where each circle represents an NMOS transistor controlled by the corresponding input variable.

Each path from the root of the stack to $S$ represents a minterm and each path from the root to $S$ represents a maxterm. It can be observed from Fig. 14 that the SAPTL implementation of XOR gates is very straightforward. By increasing the complexity of the stack, in this case increasing the number of inputs to the XOR gate, the sense amplifier and driver overhead per input can be reduced, at the expense of decreased performance. This can be seen in Fig. 15, where the energy and delay of a 6 input and 16-input SAPTL XOR gate are compared to their static CM OS equivalents. With the same $V_{T}$ (equal to low$V_{T}$ ), SAPTL reduces energy below MEP of CMOS due to longer stacks (higher effective $V_{T}$ ) and lower leakage.

The capability of SAPTL to decouple $I_{\text {Leakage }}$ and $V_{T, \text { stack }}$ is illustrated using a self-timed 64-byte parallel CRC16 generator (as used in error detection). The threshold voltages of the pass-transistors (implemented using low- $V_{T}$ devices) are reduced using varying degrees of forward body biasing. The simulated results are shown in Fig. 16 with supply voltage and activity as independent parameters. The simulation results show that the overall circuit delay can be reduced with almost no impact on energy even at low activity factors such as $\alpha=1 \%$. These results are constrained by the limited effectiveness of body biasing as a means to control $V_{T, \text { stack. }}$ The availability of devices with even lower threshold would be desirable as it would increase the effectiveness of SAPTL for energy reduction.

As can be seen in Fig. 16, the performance improvement through body biasing is more prominent at the higher supply voltage $\left(V_{D D}=0.5 \mathrm{~V}>V_{T}\right)$, at which the delay of the stack dominates the total delay. At lower supply voltages $\left(V_{D D}=0.3 \mathrm{~V} \approx V_{T}\right)$, the delay of the sense amplifier as well as the hand-shaking circuitry [15] dominates since it is near the edge of subthreshold operation, limiting the performance gains obtainable through reduction of the $V_{T, \text { stack. }}$. Circuit- and logic-level techniques are foundation for architecture-level optimizations, which will be next discussed in Section VI.

\section{ARCHITECTURAL OPTIMIZATION}

Just as parallelism showed to be effective for energy reduction around MDP, time-multiplexing is best suited for performance increase around MEP. Architectural

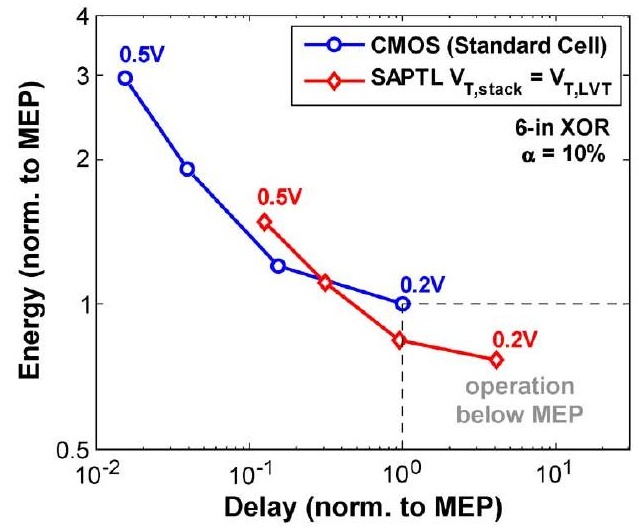

(a)

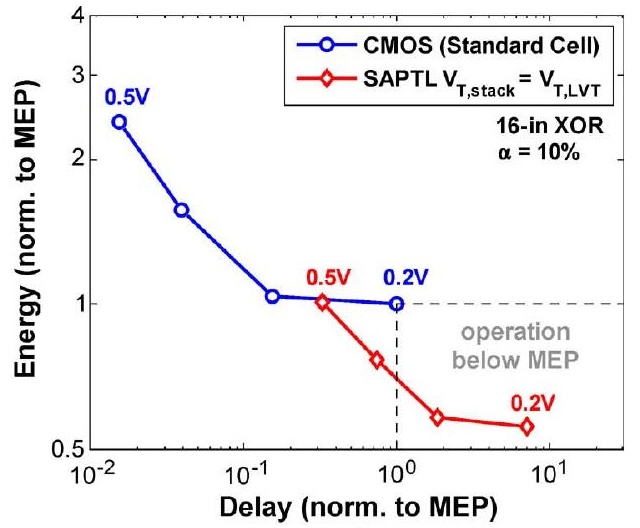

(b)

Fig. 15. Energy-delay characteristics of SAPTL designs: (a) 6-input XOR, (b) 16-input XOR. The plots show operation below MEP of static CMOS designs. 
Fig. 14. A 4-input SAPTL XOR showing the pass transistor stack structure where each circle represents an NMOS transistor controlled by the corresponding input variable.

Each path from the root of the stack to $S$ represents a minterm and each path from the root to $S$ represents a maxterm. It can be observed from Fig. 14 that the SAPTL implementation of XOR gates is very straightforward. By increasing the complexity of the stack, in this case increasing the number of inputs to the XOR gate, the sense amplifier and driver overhead per input can be reduced, at the expense of decreased performance. This can be seen in Fig. 15, where the energy and delay of a 6 input and 16-input SAPTL XOR gate are compared to their static CM OS equivalents. With the same $V_{T}$ (equal to low$V_{T}$ ), SAPTL reduces energy below MEP of CMOS due to longer stacks (higher effective $V_{T}$ ) and lower leakage.

The capability of SAPTL to decouple $I_{\text {Leakage }}$ and $V_{T, \text { stack }}$ is illustrated using a self-timed 64-byte parallel CRC16 generator (as used in error detection). The threshold voltages of the pass-transistors (implemented using low- $V_{T}$ devices) are reduced using varying degrees of forward body biasing. The simulated results are shown in Fig. 16 with supply voltage and activity as independent parameters. The simulation results show that the overall circuit delay can be reduced with almost no impact on energy even at low activity factors such as $\alpha=1 \%$. These results are constrained by the limited effectiveness of body biasing as a means to control $V_{T, \text { stack. }}$ The availability of devices with even lower threshold would be desirable as it would increase the effectiveness of SAPTL for energy reduction.

As can be seen in Fig. 16, the performance improvement through body biasing is more prominent at the higher supply voltage $\left(V_{D D}=0.5 \mathrm{~V}>V_{T}\right)$, at which the delay of the stack dominates the total delay. At lower supply voltages $\left(V_{D D}=0.3 \mathrm{~V} \approx V_{T}\right)$, the delay of the sense amplifier as well as the hand-shaking circuitry [15] dominates since it is near the edge of subthreshold operation, limiting the performance gains obtainable through reduction of the $V_{T, \text { stack. }}$. Circuit- and logic-level techniques are foundation for architecture-level optimizations, which will be next discussed in Section VI.

\section{ARCHITECTURAL OPTIMIZATION}

Just as parallelism showed to be effective for energy reduction around MDP, time-multiplexing is best suited for performance increase around MEP. Architectural

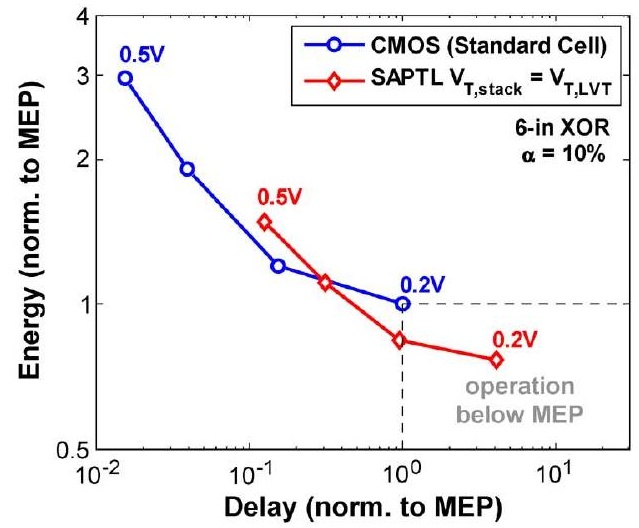

(a)

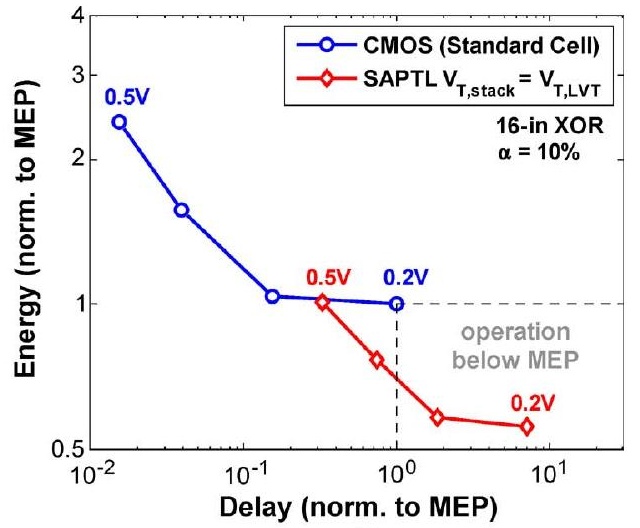

(b)

Fig. 15. Energy-delay characteristics of SAPTL designs: (a) 6-input XOR, (b) 16-input XOR. The plots show operation below MEP of static CMOS designs. 
Fig. 14. A 4-input SAPTL XOR showing the pass transistor stack structure where each circle represents an NMOS transistor controlled by the corresponding input variable.

Each path from the root of the stack to $S$ represents a minterm and each path from the root to $S$ represents a maxterm. It can be observed from Fig. 14 that the SAPTL implementation of XOR gates is very straightforward. By increasing the complexity of the stack, in this case increasing the number of inputs to the XOR gate, the sense amplifier and driver overhead per input can be reduced, at the expense of decreased performance. This can be seen in Fig. 15, where the energy and delay of a 6 input and 16-input SAPTL XOR gate are compared to their static CM OS equivalents. With the same $V_{T}$ (equal to low$V_{T}$ ), SAPTL reduces energy below MEP of CMOS due to longer stacks (higher effective $V_{T}$ ) and lower leakage.

The capability of SAPTL to decouple $I_{\text {Leakage }}$ and $V_{T, \text { stack }}$ is illustrated using a self-timed 64-byte parallel CRC16 generator (as used in error detection). The threshold voltages of the pass-transistors (implemented using low- $V_{T}$ devices) are reduced using varying degrees of forward body biasing. The simulated results are shown in Fig. 16 with supply voltage and activity as independent parameters. The simulation results show that the overall circuit delay can be reduced with almost no impact on energy even at low activity factors such as $\alpha=1 \%$. These results are constrained by the limited effectiveness of body biasing as a means to control $V_{T, \text { stack. }}$ The availability of devices with even lower threshold would be desirable as it would increase the effectiveness of SAPTL for energy reduction.

As can be seen in Fig. 16, the performance improvement through body biasing is more prominent at the higher supply voltage $\left(V_{D D}=0.5 \mathrm{~V}>V_{T}\right)$, at which the delay of the stack dominates the total delay. At lower supply voltages $\left(V_{D D}=0.3 \mathrm{~V} \approx V_{T}\right)$, the delay of the sense amplifier as well as the hand-shaking circuitry [15] dominates since it is near the edge of subthreshold operation, limiting the performance gains obtainable through reduction of the $V_{T, \text { stack. }}$. Circuit- and logic-level techniques are foundation for architecture-level optimizations, which will be next discussed in Section VI.

\section{ARCHITECTURAL OPTIMIZATION}

Just as parallelism showed to be effective for energy reduction around MDP, time-multiplexing is best suited for performance increase around MEP. Architectural

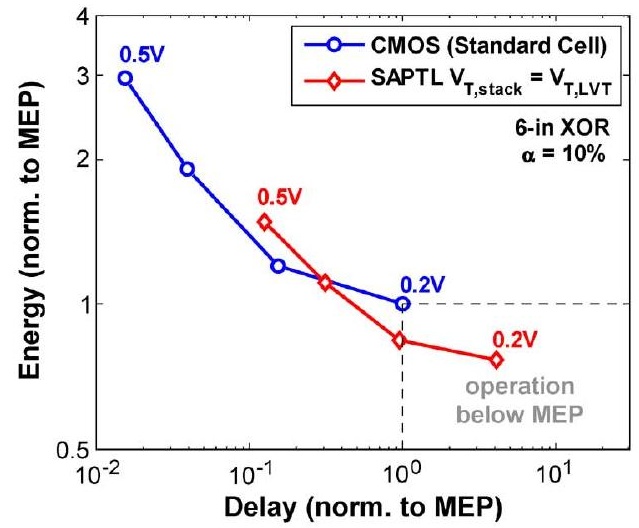

(a)

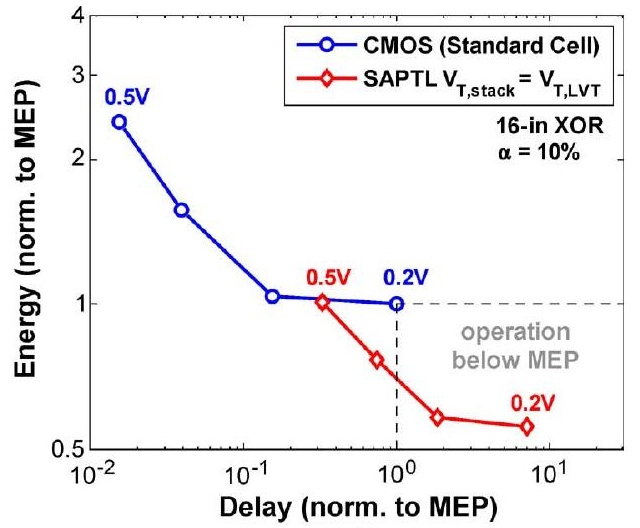

(b)

Fig. 15. Energy-delay characteristics of SAPTL designs: (a) 6-input XOR, (b) 16-input XOR. The plots show operation below MEP of static CMOS designs. 
Fig. 14. A 4-input SAPTL XOR showing the pass transistor stack structure where each circle represents an NMOS transistor controlled by the corresponding input variable.

Each path from the root of the stack to $S$ represents a minterm and each path from the root to $S$ represents a maxterm. It can be observed from Fig. 14 that the SAPTL implementation of XOR gates is very straightforward. By increasing the complexity of the stack, in this case increasing the number of inputs to the XOR gate, the sense amplifier and driver overhead per input can be reduced, at the expense of decreased performance. This can be seen in Fig. 15, where the energy and delay of a 6 input and 16-input SAPTL XOR gate are compared to their static CM OS equivalents. With the same $V_{T}$ (equal to low$V_{T}$ ), SAPTL reduces energy below MEP of CMOS due to longer stacks (higher effective $V_{T}$ ) and lower leakage.

The capability of SAPTL to decouple $I_{\text {Leakage }}$ and $V_{T, \text { stack }}$ is illustrated using a self-timed 64-byte parallel CRC16 generator (as used in error detection). The threshold voltages of the pass-transistors (implemented using low- $V_{T}$ devices) are reduced using varying degrees of forward body biasing. The simulated results are shown in Fig. 16 with supply voltage and activity as independent parameters. The simulation results show that the overall circuit delay can be reduced with almost no impact on energy even at low activity factors such as $\alpha=1 \%$. These results are constrained by the limited effectiveness of body biasing as a means to control $V_{T, \text { stack. }}$ The availability of devices with even lower threshold would be desirable as it would increase the effectiveness of SAPTL for energy reduction.

As can be seen in Fig. 16, the performance improvement through body biasing is more prominent at the higher supply voltage $\left(V_{D D}=0.5 \mathrm{~V}>V_{T}\right)$, at which the delay of the stack dominates the total delay. At lower supply voltages $\left(V_{D D}=0.3 \mathrm{~V} \approx V_{T}\right)$, the delay of the sense amplifier as well as the hand-shaking circuitry [15] dominates since it is near the edge of subthreshold operation, limiting the performance gains obtainable through reduction of the $V_{T, \text { stack. }}$. Circuit- and logic-level techniques are foundation for architecture-level optimizations, which will be next discussed in Section VI.

\section{ARCHITECTURAL OPTIMIZATION}

Just as parallelism showed to be effective for energy reduction around MDP, time-multiplexing is best suited for performance increase around MEP. Architectural

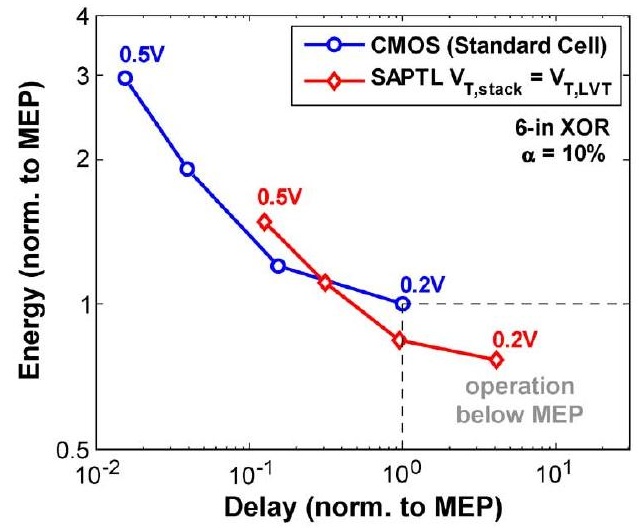

(a)

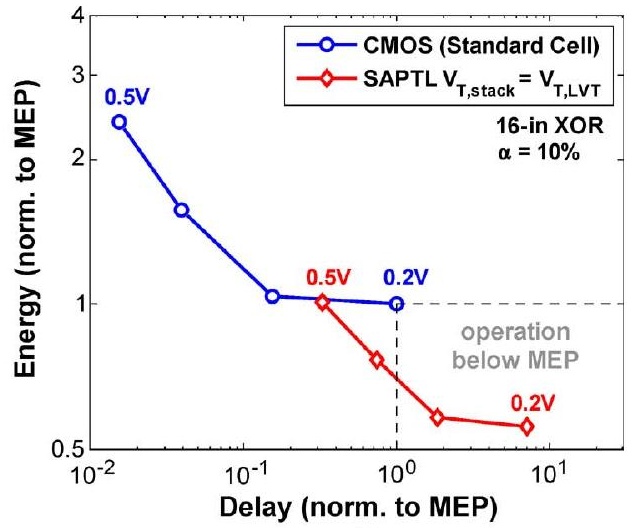

(b)

Fig. 15. Energy-delay characteristics of SAPTL designs: (a) 6-input XOR, (b) 16-input XOR. The plots show operation below MEP of static CMOS designs. 
Fig. 14. A 4-input SAPTL XOR showing the pass transistor stack structure where each circle represents an NMOS transistor controlled by the corresponding input variable.

Each path from the root of the stack to $S$ represents a minterm and each path from the root to $S$ represents a maxterm. It can be observed from Fig. 14 that the SAPTL implementation of XOR gates is very straightforward. By increasing the complexity of the stack, in this case increasing the number of inputs to the XOR gate, the sense amplifier and driver overhead per input can be reduced, at the expense of decreased performance. This can be seen in Fig. 15, where the energy and delay of a 6 input and 16-input SAPTL XOR gate are compared to their static CM OS equivalents. With the same $V_{T}$ (equal to low$V_{T}$ ), SAPTL reduces energy below MEP of CMOS due to longer stacks (higher effective $V_{T}$ ) and lower leakage.

The capability of SAPTL to decouple $I_{\text {Leakage }}$ and $V_{T, \text { stack }}$ is illustrated using a self-timed 64-byte parallel CRC16 generator (as used in error detection). The threshold voltages of the pass-transistors (implemented using low- $V_{T}$ devices) are reduced using varying degrees of forward body biasing. The simulated results are shown in Fig. 16 with supply voltage and activity as independent parameters. The simulation results show that the overall circuit delay can be reduced with almost no impact on energy even at low activity factors such as $\alpha=1 \%$. These results are constrained by the limited effectiveness of body biasing as a means to control $V_{T, \text { stack. }}$ The availability of devices with even lower threshold would be desirable as it would increase the effectiveness of SAPTL for energy reduction.

As can be seen in Fig. 16, the performance improvement through body biasing is more prominent at the higher supply voltage $\left(V_{D D}=0.5 \mathrm{~V}>V_{T}\right)$, at which the delay of the stack dominates the total delay. At lower supply voltages $\left(V_{D D}=0.3 \mathrm{~V} \approx V_{T}\right)$, the delay of the sense amplifier as well as the hand-shaking circuitry [15] dominates since it is near the edge of subthreshold operation, limiting the performance gains obtainable through reduction of the $V_{T, \text { stack. }}$. Circuit- and logic-level techniques are foundation for architecture-level optimizations, which will be next discussed in Section VI.

\section{ARCHITECTURAL OPTIMIZATION}

Just as parallelism showed to be effective for energy reduction around MDP, time-multiplexing is best suited for performance increase around MEP. Architectural

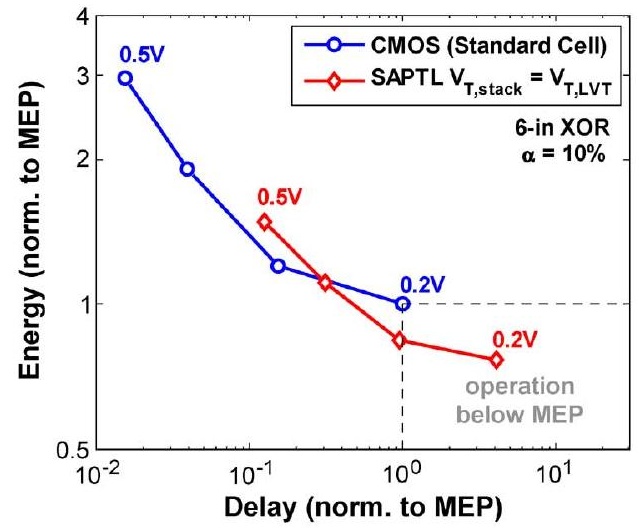

(a)

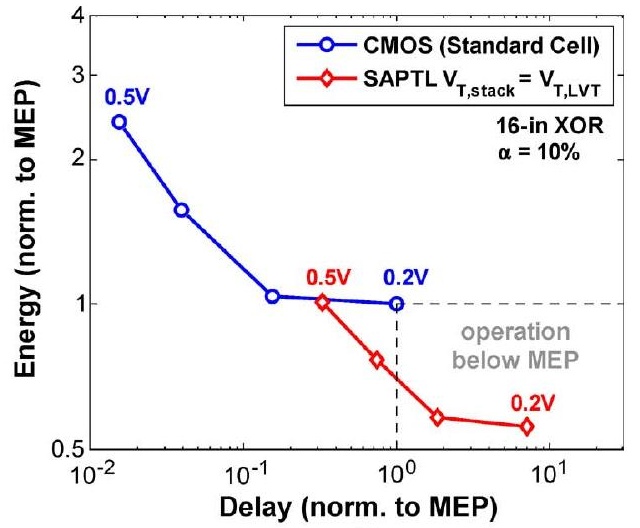

(b)

Fig. 15. Energy-delay characteristics of SAPTL designs: (a) 6-input XOR, (b) 16-input XOR. The plots show operation below MEP of static CMOS designs. 
Fig. 14. A 4-input SAPTL XOR showing the pass transistor stack structure where each circle represents an NMOS transistor controlled by the corresponding input variable.

Each path from the root of the stack to $S$ represents a minterm and each path from the root to $S$ represents a maxterm. It can be observed from Fig. 14 that the SAPTL implementation of XOR gates is very straightforward. By increasing the complexity of the stack, in this case increasing the number of inputs to the XOR gate, the sense amplifier and driver overhead per input can be reduced, at the expense of decreased performance. This can be seen in Fig. 15, where the energy and delay of a 6 input and 16-input SAPTL XOR gate are compared to their static CM OS equivalents. With the same $V_{T}$ (equal to low$V_{T}$ ), SAPTL reduces energy below MEP of CMOS due to longer stacks (higher effective $V_{T}$ ) and lower leakage.

The capability of SAPTL to decouple $I_{\text {Leakage }}$ and $V_{T, \text { stack }}$ is illustrated using a self-timed 64-byte parallel CRC16 generator (as used in error detection). The threshold voltages of the pass-transistors (implemented using low- $V_{T}$ devices) are reduced using varying degrees of forward body biasing. The simulated results are shown in Fig. 16 with supply voltage and activity as independent parameters. The simulation results show that the overall circuit delay can be reduced with almost no impact on energy even at low activity factors such as $\alpha=1 \%$. These results are constrained by the limited effectiveness of body biasing as a means to control $V_{T, \text { stack. }}$ The availability of devices with even lower threshold would be desirable as it would increase the effectiveness of SAPTL for energy reduction.

As can be seen in Fig. 16, the performance improvement through body biasing is more prominent at the higher supply voltage $\left(V_{D D}=0.5 \mathrm{~V}>V_{T}\right)$, at which the delay of the stack dominates the total delay. At lower supply voltages $\left(V_{D D}=0.3 \mathrm{~V} \approx V_{T}\right)$, the delay of the sense amplifier as well as the hand-shaking circuitry [15] dominates since it is near the edge of subthreshold operation, limiting the performance gains obtainable through reduction of the $V_{T, \text { stack. }}$. Circuit- and logic-level techniques are foundation for architecture-level optimizations, which will be next discussed in Section VI.

\section{ARCHITECTURAL OPTIMIZATION}

Just as parallelism showed to be effective for energy reduction around MDP, time-multiplexing is best suited for performance increase around MEP. Architectural

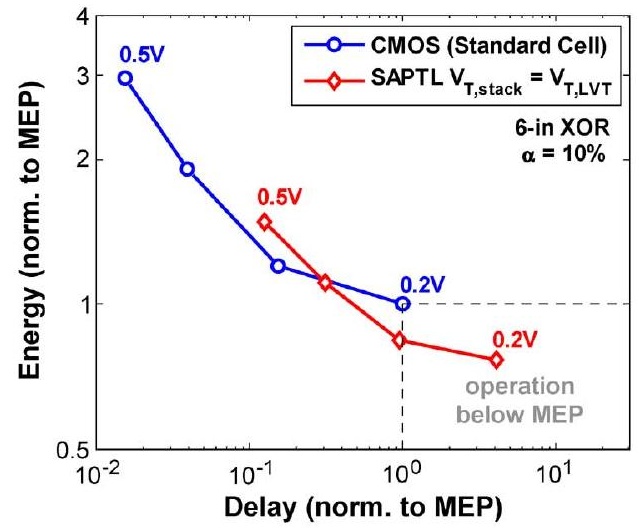

(a)

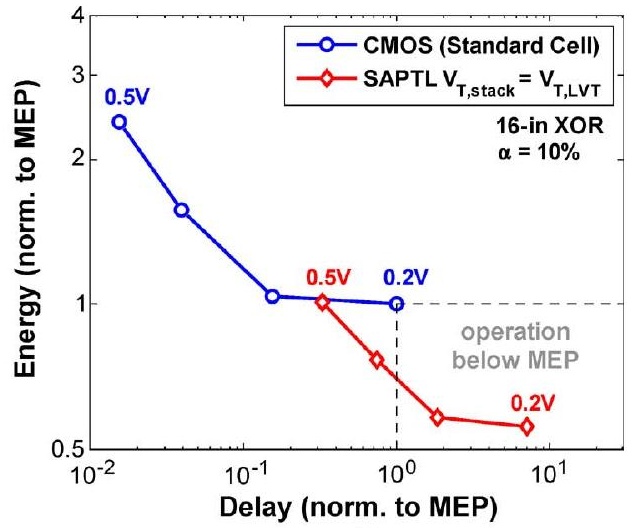

(b)

Fig. 15. Energy-delay characteristics of SAPTL designs: (a) 6-input XOR, (b) 16-input XOR. The plots show operation below MEP of static CMOS designs. 
Fig. 14. A 4-input SAPTL XOR showing the pass transistor stack structure where each circle represents an NMOS transistor controlled by the corresponding input variable.

Each path from the root of the stack to $S$ represents a minterm and each path from the root to $S$ represents a maxterm. It can be observed from Fig. 14 that the SAPTL implementation of XOR gates is very straightforward. By increasing the complexity of the stack, in this case increasing the number of inputs to the XOR gate, the sense amplifier and driver overhead per input can be reduced, at the expense of decreased performance. This can be seen in Fig. 15, where the energy and delay of a 6 input and 16-input SAPTL XOR gate are compared to their static CM OS equivalents. With the same $V_{T}$ (equal to low$V_{T}$ ), SAPTL reduces energy below MEP of CMOS due to longer stacks (higher effective $V_{T}$ ) and lower leakage.

The capability of SAPTL to decouple $I_{\text {Leakage }}$ and $V_{T, \text { stack }}$ is illustrated using a self-timed 64-byte parallel CRC16 generator (as used in error detection). The threshold voltages of the pass-transistors (implemented using low- $V_{T}$ devices) are reduced using varying degrees of forward body biasing. The simulated results are shown in Fig. 16 with supply voltage and activity as independent parameters. The simulation results show that the overall circuit delay can be reduced with almost no impact on energy even at low activity factors such as $\alpha=1 \%$. These results are constrained by the limited effectiveness of body biasing as a means to control $V_{T, \text { stack. }}$ The availability of devices with even lower threshold would be desirable as it would increase the effectiveness of SAPTL for energy reduction.

As can be seen in Fig. 16, the performance improvement through body biasing is more prominent at the higher supply voltage $\left(V_{D D}=0.5 \mathrm{~V}>V_{T}\right)$, at which the delay of the stack dominates the total delay. At lower supply voltages $\left(V_{D D}=0.3 \mathrm{~V} \approx V_{T}\right)$, the delay of the sense amplifier as well as the hand-shaking circuitry [15] dominates since it is near the edge of subthreshold operation, limiting the performance gains obtainable through reduction of the $V_{T, \text { stack. }}$. Circuit- and logic-level techniques are foundation for architecture-level optimizations, which will be next discussed in Section VI.

\section{ARCHITECTURAL OPTIMIZATION}

Just as parallelism showed to be effective for energy reduction around MDP, time-multiplexing is best suited for performance increase around MEP. Architectural

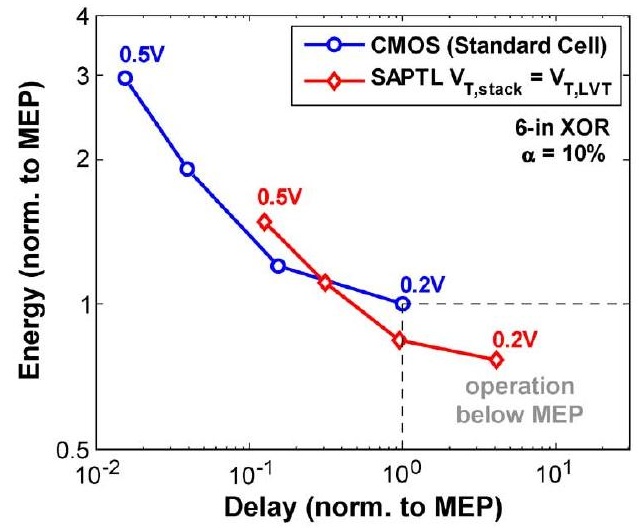

(a)

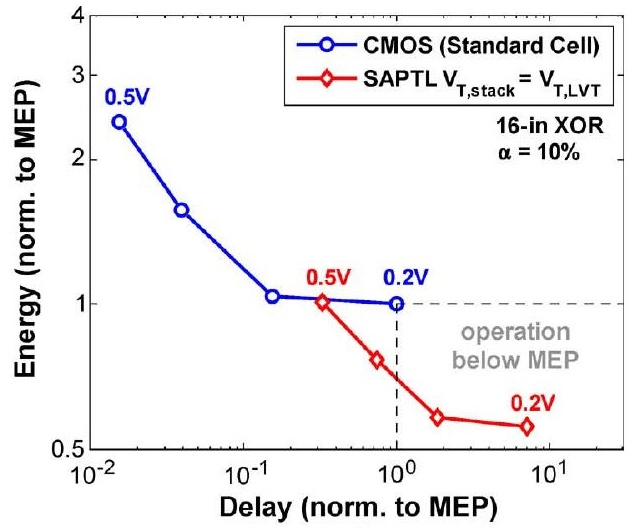

(b)

Fig. 15. Energy-delay characteristics of SAPTL designs: (a) 6-input XOR, (b) 16-input XOR. The plots show operation below MEP of static CMOS designs. 
Fig. 14. A 4-input SAPTL XOR showing the pass transistor stack structure where each circle represents an NMOS transistor controlled by the corresponding input variable.

Each path from the root of the stack to $S$ represents a minterm and each path from the root to $S$ represents a maxterm. It can be observed from Fig. 14 that the SAPTL implementation of XOR gates is very straightforward. By increasing the complexity of the stack, in this case increasing the number of inputs to the XOR gate, the sense amplifier and driver overhead per input can be reduced, at the expense of decreased performance. This can be seen in Fig. 15, where the energy and delay of a 6 input and 16-input SAPTL XOR gate are compared to their static CM OS equivalents. With the same $V_{T}$ (equal to low$V_{T}$ ), SAPTL reduces energy below MEP of CMOS due to longer stacks (higher effective $V_{T}$ ) and lower leakage.

The capability of SAPTL to decouple $I_{\text {Leakage }}$ and $V_{T, \text { stack }}$ is illustrated using a self-timed 64-byte parallel CRC16 generator (as used in error detection). The threshold voltages of the pass-transistors (implemented using low- $V_{T}$ devices) are reduced using varying degrees of forward body biasing. The simulated results are shown in Fig. 16 with supply voltage and activity as independent parameters. The simulation results show that the overall circuit delay can be reduced with almost no impact on energy even at low activity factors such as $\alpha=1 \%$. These results are constrained by the limited effectiveness of body biasing as a means to control $V_{T, \text { stack. }}$ The availability of devices with even lower threshold would be desirable as it would increase the effectiveness of SAPTL for energy reduction.

As can be seen in Fig. 16, the performance improvement through body biasing is more prominent at the higher supply voltage $\left(V_{D D}=0.5 \mathrm{~V}>V_{T}\right)$, at which the delay of the stack dominates the total delay. At lower supply voltages $\left(V_{D D}=0.3 \mathrm{~V} \approx V_{T}\right)$, the delay of the sense amplifier as well as the hand-shaking circuitry [15] dominates since it is near the edge of subthreshold operation, limiting the performance gains obtainable through reduction of the $V_{T, \text { stack. }}$. Circuit- and logic-level techniques are foundation for architecture-level optimizations, which will be next discussed in Section VI.

\section{ARCHITECTURAL OPTIMIZATION}

Just as parallelism showed to be effective for energy reduction around MDP, time-multiplexing is best suited for performance increase around MEP. Architectural

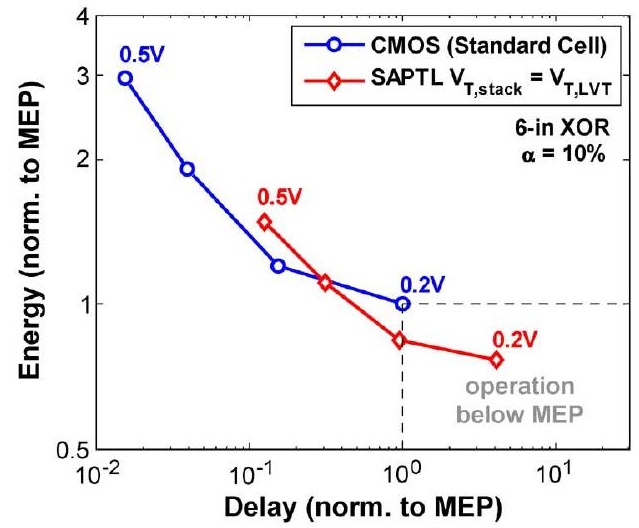

(a)

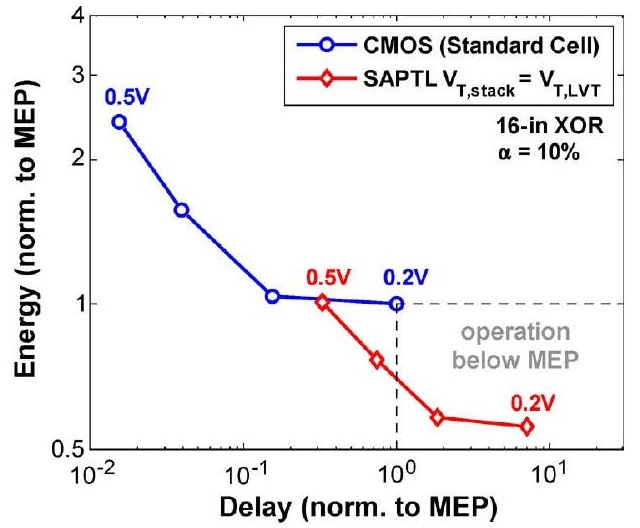

(b)

Fig. 15. Energy-delay characteristics of SAPTL designs: (a) 6-input XOR, (b) 16-input XOR. The plots show operation below MEP of static CMOS designs. 
Fig. 14. A 4-input SAPTL XOR showing the pass transistor stack structure where each circle represents an NMOS transistor controlled by the corresponding input variable.

Each path from the root of the stack to $S$ represents a minterm and each path from the root to $S$ represents a maxterm. It can be observed from Fig. 14 that the SAPTL implementation of XOR gates is very straightforward. By increasing the complexity of the stack, in this case increasing the number of inputs to the XOR gate, the sense amplifier and driver overhead per input can be reduced, at the expense of decreased performance. This can be seen in Fig. 15, where the energy and delay of a 6 input and 16-input SAPTL XOR gate are compared to their static CM OS equivalents. With the same $V_{T}$ (equal to low$V_{T}$ ), SAPTL reduces energy below MEP of CMOS due to longer stacks (higher effective $V_{T}$ ) and lower leakage.

The capability of SAPTL to decouple $I_{\text {Leakage }}$ and $V_{T, \text { stack }}$ is illustrated using a self-timed 64-byte parallel CRC16 generator (as used in error detection). The threshold voltages of the pass-transistors (implemented using low- $V_{T}$ devices) are reduced using varying degrees of forward body biasing. The simulated results are shown in Fig. 16 with supply voltage and activity as independent parameters. The simulation results show that the overall circuit delay can be reduced with almost no impact on energy even at low activity factors such as $\alpha=1 \%$. These results are constrained by the limited effectiveness of body biasing as a means to control $V_{T, \text { stack. }}$ The availability of devices with even lower threshold would be desirable as it would increase the effectiveness of SAPTL for energy reduction.

As can be seen in Fig. 16, the performance improvement through body biasing is more prominent at the higher supply voltage $\left(V_{D D}=0.5 \mathrm{~V}>V_{T}\right)$, at which the delay of the stack dominates the total delay. At lower supply voltages $\left(V_{D D}=0.3 \mathrm{~V} \approx V_{T}\right)$, the delay of the sense amplifier as well as the hand-shaking circuitry [15] dominates since it is near the edge of subthreshold operation, limiting the performance gains obtainable through reduction of the $V_{T, \text { stack. }}$. Circuit- and logic-level techniques are foundation for architecture-level optimizations, which will be next discussed in Section VI.

\section{ARCHITECTURAL OPTIMIZATION}

Just as parallelism showed to be effective for energy reduction around MDP, time-multiplexing is best suited for performance increase around MEP. Architectural

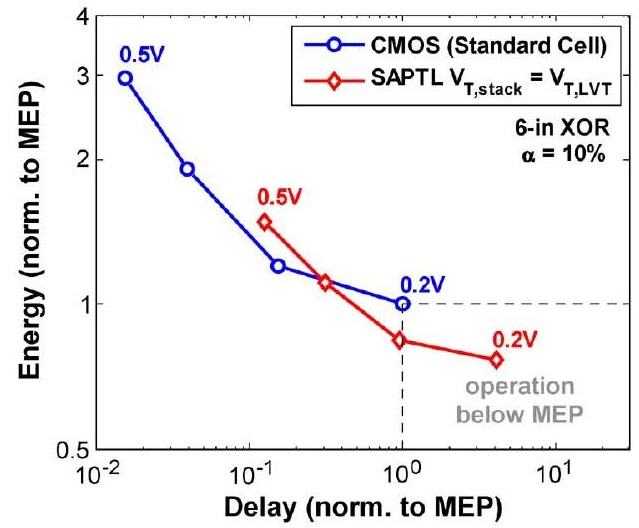

(a)

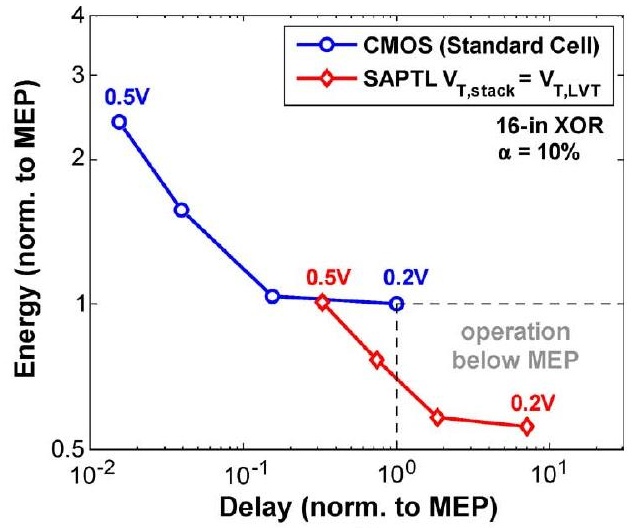

(b)

Fig. 15. Energy-delay characteristics of SAPTL designs: (a) 6-input XOR, (b) 16-input XOR. The plots show operation below MEP of static CMOS designs. 